\title{
Delayed processing in accented speech: Evidence from a mouse-tracking experiment
}

June 12, 2019

\begin{abstract}
Previous work shows that listeners use the phonetic signal to predict upcoming information, including adaptation to accent-specific phonological structures. This study assesses whether this response is optimal by studying the time course and specificity of adaptation as native French listeners are presented with Englishaccented French samples in a cross-modal two-alternative forced choice task. There were four types of trials, depending on whether the spoken target word contained a sound replaced in English-accented speech with a different phoneme; and whether this pronunciation rendered the choice temporarily ambiguous or not. If listeners follow an optimal strategy, one predicts an interaction between both terms, with slower responses for words with replaced phonemes only when the replacement rendered the target temporarily ambiguous. Instead, we found main effects of both factors. Thus, it appears that adult listeners did not adopt the strategy that would have been informationally optimal. This result could be due to prior experience with the accent employed, and specific difficulties with replaced phonemes, despite knowledge of the accent. We discuss results in the broader context of accent perception research, bearing on the (speaker- and contrast-)specificity of listener adaptation as well as the variability in results potentially due to the task, accent, and materials employed.
\end{abstract}

Keywords: redictability; accented speech; mouse-tracking

\section{Introduction}

Listeners can use phonetic information to predict upcoming words as the signal unfolds. When (the preceding context and) phonetic information does not immediately disambiguate two possible targets (e.g., "candle" and "candy" which both begin with [kand] and only differ at the fifth phoneme) a listener will be delayed relative to when they can disambiguate early (e.g., "candle" and "towel" which differ at the first phoneme) (Tanenhaus, Spivey-Knowlton, Eberhard, \& Sedivy, 1995; Allopenna, Magnuson, \& Tanenhaus, 1998). Listeners further incorporate speaker- and accent-specific information when developing expectations and/or processing incoming speech, leading them to adjust their expectations in the face of variation from their standard (Bradlow \& Bent, 2008; Janse \& Adank, 2012). This previous experimental research suggests 
that some adaptation happens relatively fast, after just a few minutes of hearing accented sentences (Clarke \& Garrett, 2004), or even after hearing a handful of tokens unambiguously cuing a pronunciation shift (Dahan, Drucker, \& Scarborough, 2008), and by and large appears to involve generalization beyond the specific words heard in the exposure, including to sound categories that are reasonable extensions for the accent-specific rules (Eisner, Melinger, \& Weber, 2013). But is this adaptation procedure optimal? That is, do listeners make all and only the expectation changes that allow them to optimize their responses? In this paper, we report on an experiment building on accents leading to replacement, which is an ideal situation for answering that question, as we explain next.

Most previous work has investigated cases in which the target is slightly shifted (e.g., the boundary between /s/ and /f/ is set at a different point, Norris, McQueen, \& Cutler, 2003). However, cases of "replacement", where surface implementation spans into a separate phoneme's typical surface form, are particularly useful to study the optimality of expectation adaptations, because, unlike boundary shifts, replacements can effectively create homophones. For example, French-native speakers contrast the words dessous ("beneath") and dessus ("above") by the vowel ([u] and [y] respectively), but since English only has [u], both words are typically pronounced as [dəsu] in English-accented speech.

In this paper, we presented listeners with a two-alternative intermodal matching task. Participants saw two images side by side, and were instructed by an accented speaker to click on one of the two images. There were four kinds of trials, resulting from the crossing of two factors: whether the target word contained a replaced phoneme in the speaker's accent or not; and whether this sound would have disambiguated the target word or not (i.e., the target could have been identified by other material earlier in the word, because the spoken word onset unambiguously pointed to one of the words). If accent adaptation optimizes choice behavior, listeners should learn to delay their decision for replacements leading to temporarily ambiguous targets, but not for replacements that do not lead to ambiguous targets. To examine processing of the speech signal as it unfolds, we used a mouse-tracking procedure in addition to collecting reaction times. Mouse-tracking allows continuous tracking of information integration in a way that ballistic eye movements do not permit. To our knowledge, this application remains rather novel, as only a handful of studies have incorporated mousetracking for the study of speech perception decisions (Spivey, Grosjean, \& Knoblich, 2005; Farmer, Liu, Mehta, \& Zevin, 2009; Krestar, Incera, \& McLennan, 2013).

\section{Methods}

This study was pre-registered and materials are hosted on the Open Science Framework (OSF; XXX [hidden for review]). Some initial hypotheses have been revised, as explained in a separate document on the same site due to space constraints. 


\subsection{Participants}

The final sample consisted of 30 participants. This number was decided on prior to data collection based on a power analysis with six pilot participants (none of whom are included in the final sample). Included participants were native speakers of French and not fluent in any other language from birth. Participants had an average English proficiency of 7 (standard deviation $(\mathrm{SD})=1.8$ ) on a scale of 0 (none) to 10 (perfect). For speaking English, 10 participants said they speak English more than once a week, 12 between once a week and once a month, 6 less than once a month, and 2 never. For hearing English, 14 participants said they hear English more than once a week, 5 between once a week and once a month, 7 less than once a month, and 4 never. An additional 9 participants were dropped due to being bilingual from before the age of six $(\mathrm{N}=6)$, or not reaching a minimum number of viable trials $(\mathrm{N}=3)$.

\subsection{Materials}

All stimuli are available in the online supplementary materials. Spoken stimuli were 4 carrier phrases and 40 words spliced from materials produced by one native speaker of British English, speaking accented French. Carrier phrases had the structure Cliquez. l'image pour ("Click the picture of"), and did not contain any replaced phonemes. Words were required to have a frequency of at least 0.5 per-million. Words could be of two types: 1) no phoneme replacement (where the accent did not result in a replaced phoneme), or 2) phoneme replacement (where the accent caused one phoneme in French, not present in English, to be replaced with another phoneme present in both languages). Both consonant and vowel replacements were included. The consonant error meant replacing negative voice onset time (VOT) with short-lag VOT $(/ \mathrm{b} / \rightarrow[\mathrm{p}]$, $/ \mathrm{d} / \rightarrow[\mathrm{t}], / \mathrm{g} / \rightarrow[\mathrm{k}])$. Vowel errors were: 1$)$ producing an oral vowel and a nasal instead

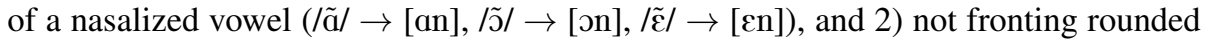
vowels $(/ \mathrm{y} / \rightarrow[\mathrm{u}], / \varnothing / \rightarrow[\mathrm{o}])$. For the consonant errors, the first $\mathrm{C}$ was the consonant under investigation. For the vowel errors, the first $\mathrm{V}$ was the vowel under investigation. All words were chosen from the Lexique 3.80 database (New, Pallier, Ferrand, $\&$ Matos, 2001). For more information about how the audio files were constructed see our online supplementary materials.

Items were paired in trials varying in ambiguity, and which we will call "filler" and "test" trials. In filler trials, the target and the competitor words were not phonologically related. In test trials, the target and the competitor words differed by the first or second phoneme in native speech, but did not disambiguate until later in the word in Englishaccented French. Items were combined such that every word appeared as target and competitor, in filler and test trials, over 4 different blocks, for a total of 160 trials. See Table 1 for 4 sample trials.

Pictures representing each word were taken from a website of free, creative commons photos (http://www.morguefile.com/). For some of them, photos were edited to point out a specific part of the image, or several photos were shown together to convey the concept. For one word, baluchon ("bundle"), a suitable picture could not be found and a line drawing was created in Keynote. 
Table 1: Example trials for ambiguity (filler, test) x phoneme replacement (no replacement, replacement).

\begin{tabular}{|c|c|c|c|}
\hline Ambiguity & $\begin{array}{l}\text { Replacement } \\
\text { in Target }\end{array}$ & Target & Competitor \\
\hline Filler & No replacement & palissade & lendemain \\
\hline Filler & Replacement & lendemain & palissade \\
\hline Test & No replacement & palissade & baratin \\
\hline Test & Replacement & lendemain & laverie \\
\hline
\end{tabular}

\subsection{Procedure}

The experiment was conducted in French. Participants began with a familiarization phase during which they saw all the images one by one, with the corresponding word (written orthographically) written immediately below the image. No auditory stimuli were used during this phase. After familiarization, participants were told to alert the experimenter to have the second part of the experiment explained to them. The experimenter then told participants that they would hear sentences and be presented with two images; they should click on the image that had been named. All instructions were displayed orthographically as well as explained orally. In every trial, the carrier phrase was played automatically; however, the sound was paused after the carrier phrase and listeners had to begin to move the mouse up towards the images for the target word to be played. Participants were asked to move the mouse and make their decision as quickly as possible. The trial timed out after two seconds (with a message to go faster), so as to encourage rapid responses. After each trial, the participant clicked on a spot at the bottom of the screen so as to physically reset the mouse for the next trial.

After completing the experiment, participants filled out a brief language questionnaire. The questionnaire included questions regarding exposure to accents, both of the test accent and accented speech in general as well as proficiency in English. All parts of the experiment were administered in MATLAB on a MacBook Air. An Apple Magic Mouse was used with mouse speed set to maximum.

\subsection{Participant exclusion criteria}

Participants and data were excluded following a series of requirements, determined before inspecting results. First, participants were required to have at least 5 valid trials per cell, block $(1$ through 4$) \times$ ambiguity (filler, test $) \times$ phoneme replacement (no replacement, replacement), to be included into the analyses (see supplementary materials for justification). For all remaining participants, all trials incorrectly answered (either due to the trial timing out or an incorrect response) were dropped. Any trial with a reaction time two standard deviations above or below the mean for a given participant for a given cell of block $(1$ through 4$) \times$ ambiguity (filler, test $) \times$ phoneme replacement (no replacement, replacement) was dropped. This resulted in a loss of 381 data points across all 30 participants ( $7.9 \%$ of the data). 


\section{Results}

To test for statistical significance, two linear mixed effects model were fit, one with reaction time as the dependent variable and one with the mouse-tracking measure as the dependent variable (see below for the description of the mouse-tracking measure). The fixed effects were ambiguity (filler, test), phoneme replacement (no replacement, replacement), trial number within block, (1 - 40), and block (1 - 4). Note, trial number was specific to a given block $(1-40)$, not the trial number relative to the entire experiment $(1-160)$. This allowed us to see if there was an interaction of trial number and block. All main effects were included as well as the two-way interaction of trial number by block and the three-way interaction of ambiguity by phoneme replacement by block. Only these interactions were included to avoid the model becoming too complex, and because these interactions were deemed most important for the hypotheses. The two categorical fixed effects were contrast coded; trial number and block were centered and coded as numeric. For the reaction time analysis, participant was included as a random intercept and as a random slope by ambiguity, uncorrelated with the random intercept. Target word nested in pair was included as a random intercept. For the mouse-tracking analysis, participant was included as a random intercept and a random slope by ambiguity and phoneme replacement, uncorrelated with the random intercept. Target word nested in pair was included as a random intercept and as a random slope by trial number uncorrelated with the random intercept. These were the maximal random effects structures that would converge following (Barr, Levy, Scheepers, \& Tily, 2013). Significance was assessed with model comparison and an alpha of 0.05 .

\subsection{Reaction times}

Figure 1 shows reaction times by block, ambiguity, phoneme replacement, and trial number. Based on the plot it appears that participants are in general slower on test items, and potentially slower on replacement items, at least for fillers.

The linear mixed effects model found a significant effect of ambiguity, with slower responses for test trials $\left[\beta=51.13, S E=8.45, \chi^{2}(1)=26.37, p<0.001\right]$, a significant effect of phoneme replacement, with slower responses for replacement targets $[\beta=$ 29.53, $\left.S E=9.88, \chi^{2}(1)=7.30, p<0.01\right]$, a significant effect of trial number, with faster responses as the trial number increased $\left[\beta=-3.21, S E=0.42, \chi^{2}(1)=57.69\right.$, $p<0.001$ ], and a significant effect of block, with faster responses as the experiment progressed $\left[\beta=-18.96, S E=3.82, \chi^{2}(1)=24.51, p<0.001\right]$. Finally, the interaction of trial number and block was significant $\left[\beta=2.27, S E=0.34, \chi^{2}(1)=44.31, p<\right.$ $0.001]$.

To better understand the interaction of trial number and block, follow-up simple regressions were run, one for each block. Trial number was significant for Block 1 , with a speeding up throughout the block $[\beta=-7.64, S E=0.88, t=-8.73, p<0.001]$, but not for any of the other blocks. 


\subsection{Mouse-tracking}

Figure 2 shows smoothed curves of mouse movements over time by block, ambiguity, and phoneme replacement. Based on the plot, it appears that participants deviate from the straight upward trajectory and move the mouse towards the correct item earlier for filler items than for test items. Also, for both filler and test items, they appear to do this earlier for no replacement items than those with a replacement.

To better quantify the "break point" (the point at which a participant makes a sharp movement up towards the correct image), for each trial $100 \mathrm{~ms}$ windows was taken starting from 0 to 100 and then moving by $10 \mathrm{~ms}$ (e.g., 0 - $100 \mathrm{~ms}, 10-110 \mathrm{~ms}$, etc.). For each window a simple linear model was run with distance from incorrect item as the dependent variable and time as the independent variable. The slopes for each regression were then extracted and the first slope greater than or equal to 1 pixel/millisecond selected. This was considered the point at which the participant made the decision to move towards the correct image. If they were unsure the slope would be near zero, and if they moved towards the incorrect image the slope would be negative. The choice of a minimum slope of 1 was based on visual inspection of the data before running the models. For the selected window, the minimum time (left side of the window) was considered to be the break point for that specific trial. With this method, 149 additional trials were lost due to the slope never attaining 1 for a given window (a total of $11.0 \%$ of the data lost including reaction time cleaning). A figure of some example trials are presented in Figure 3; the vertical line marks the break point as determined by the model for that trial. Figure 4 shows how the break point changes over time by ambiguity, phoneme replacement, and trial number.

The linear mixed effects model again found a significant effect of ambiguity, with later break points for test trials [ $\left.\beta=41.96, S E=8.44, \chi^{2}(1)=16.68, p<0.001\right]$, and a significant effect of trial number, with earlier break points as the trial number increased $\left[\beta=-1.54, S E=0.34, \chi^{2}(1)=20.86, p<0.001\right]$. The effect of phoneme replacement was only trending $\left[\beta=19.94, S E=9.83, \chi^{2}(1)=3.72, p=0.05\right]$. The main effect of block was not significant. Finally, the interaction of trial number and block was significant $\left[\beta=1.03, S E=0.27, \chi^{2}(1)=14.59, p<0.001\right]$.

To better understand the interaction of trial number and block, follow-up simple regressions were run, one for each block. Trial number was significant for Block 1, with earlier break points as the experiment progressed $[\beta=-3.69, S E=0.77, t=-4.81$, $p<0.001]$, but not for any of the other blocks.

\section{Discussion}

A main goal of our study was to test for adaptation, assuming optimal listeners. This would show minimally via a two-way interaction of ambiguity (filler, test) by phoneme replacement (no replacement, replacement) (or potentially in a three-way interaction with block, if participants' optimal behavior arose in later trials). Our results did not support this precise prediction - a matter to which we return later -, although several factors explained significant variance, as follows. We found that participants delayed decisions both for trials where the phonological forms of target and competitor were 
similar, and where the phonetic form of the target word contained a replaced sound, without interaction between the two factors, and without interaction with block.

How may the main effect of ambiguity be interpreted? This result is straightforward: When target and competitor are similar, the decision is more difficult than when there is no phonological overlap, an effect already documented, for instance, by Spivey et al. (2005) using a mouse-tracking paradigm similar to the one used here, and comparing a cohort (candle - candy) against a control (candle - picture) conditions.

How about the consistent delay when the spoken target word contained a replaced phoneme? It is important to bear in mind that it was not the mere presence of an accent. Recall that all items were accented on a phonetic level, resulting in non-standard pronunciation of many sounds, including use of the English $[\mathrm{I}]$ for the French $[\mathrm{b}]$. Instead, this main effect shows that the delay is particularly marked when the accent results in a phoneme replacement such that two French phonemes map to a single production in accented-French, and interestingly, even when the forced choice remains unambiguous in the context.

On the surface, this result appears to be at odds with reports that listeners perceptually adapt in both a productive and specific, accepting wetch as a word for a speaker that has exhibited $/ \mathrm{I} / \rightarrow[\varepsilon]$ vowel lowering, but not weetch (Maye, Aslin, \& Tanenhaus, 2008; see also Eisner et al., 2013; Kraljic, Samuel, \& Brennan, 2008 for other evidence on the specificity of effects). However, it may be inappropriate to compare our results to theirs, because they employ lexical decision tasks (where adaptation to an accent is inferred from changes in response to the question "is this a word"). In contrast, here we investigate online processing and provide listeners with no option to reject the items as non-words.

A better comparison case are studies that use continuous measures, typically eyetracking in an intermodal matching paradigm (which, incidentally, also often focus on phonemic mergers, such as devoicing rules inducing neutralization of a contrast, or replacement of the type we saw here). Our reading of such literature (to cite just two, Dahan et al., 2008; Ito \& Campbell-Kibler, 2011) is that they have typically focused on the most algid case, that of temporarily induced ambiguities with a competitor that is a plausible referent at least early on. However, these studies have not incorporated a control case like the one we have, where there is no ambiguity (that is, when the labels for the competitor and target image labels are not similar). In other words, we replicate their findings that replacement slows down decision, now using a continuous mousetracking measure rather than averages over categorical individual-level decisions. We extend their findings by showing that listeners are slowed down in their decision even in cases where no plausible alternative is present, which in our view is far from a trivial extension of that work. Importantly, our finding provides evidence in favor of exemplar-based models (e.g., Pierrehumbert, 2002), with recognition being delayed by a mismatch between the token heard and the tokens in long-term memory, and against their rule-based competitors, where evidence for replacement in one lexical item should have been productively generalized to others, and thus no processing loss should occur in unambiguous contexts. Future work should explore this effect in more detail.

This is a good segue back to the main goal of our study, to study whether adaptation was optimal. One unexpected result was the absence of interaction with block. Might this be because the adaptation was too rapid to detect statistically? Indeed, 14 out of 
the 30 participants correctly identified the accent as being from the United Kingdom. Given this prior experience they may have been able to quickly change their expectations as soon as they detected the familiar accent.

The second, and more problematic, unexpected result was the lack of significance of the predicted 2-way interaction: As mentioned previously, optimal listeners should slow down for test (i.e., ambiguous) trials with replacement more than all other combinations, particularly as the experiment proceeds. Yet we do not observe this. As explained above, the main effects of ambiguity (slower for test trials with more similar target and competitor, than filler trials, where target and competitor labels do not sound similar) and replacement (slower for trials where the target word has been mispronounced than trials where the accent did not lead to a phoneme replacement) could be explained without making reference to optimal adaptation at all. Thus, we are led to conclude that, counter to suggestions from offline lexical decision studies, either accent adaptation is not optimally implemented at all, or it may require a different type of setting than the one implemented here. If so, it may not represent a generalized speech processing strategy, but rather a task-specific response.

Overall, we would like to stress the importance of differentiating between: 1) processing difficulties due to the general presence of an accent; 2) processing difficulties where non-standard pronunciations are amiable to an accent-specific repair strategy; and 3) processing difficulties due to non-standard pronunciations that cannot be efficiently undone. In our study, we did not measure the effects of (1) nor its reduction with exposure, unlike (Bradlow \& Bent, 2008), because we did not have a control, unaccented voice. (Notice that the speeding up we observed in the first block of trials may well have been a practice effect with the task.) A host of studies demonstrate that even short exposures can result in changes in decision behavior when listeners face (2).

The most novel aspect of our results is the report of a persistent and suboptimal difficulty with (3) despite the exposure to a disambiguating context that would allow listeners to develop a better decision strategy, namely slowing down specifically for test-replacement trials. We believe the most appropriate interpretation of this result is that adaptation is not optimal. Nonetheless, we admit out that, given the diversity in design and materials across different studies, a direct comparison is difficult. Thus, future work should attempt to design an experiment that covers the whole host of factors, for instance by including four voices, one of which does not have an accent and another that has an accent which only affects the intonation and phonetic detail (to isolate factor 1); a third voice that implements a rule that can be easily repaired (factor 2); and a forth which contains a replacement like the one used here. Ideally, the experiment would be long enough that participants have time to completely adapt to the accent errors (if that is possible in the context of an experiment). This way the time course of these three factors would be effectively separated.

\section{Conclusions}

The present study is among the first to employ a mouse-tracking task to investigate the time course of lexical recognition in an intermodal matching task, a method we used to investigate whether adaptation to accented speech is optimal. Our results led 
us to conclude it is not. Additionally, we document a generalized slow-down even in the absence of plausible alternatives whenever there was a phoneme replacement, that should be investigated in future work.

\section{References}

Allopenna, P. D., Magnuson, J. S., \& Tanenhaus, M. K. (1998). Tracking the time course of spoken word recognition using eye movements: Evidence for continuous mapping models. Journal of Memory and Language, 38(4), 419 - 439. doi: http://dx.doi.org/10.1006/jmla.1997.2558

Barr, D. J., Levy, R., Scheepers, C., \& Tily, H. J. (2013, apr). Random effects structure for confirmatory hypothesis testing: Keep it maximal. Journal of Memory and Language, 68(3), 255-278. doi: 10.1016/j.jml.2012.11.001

Bradlow, A. R., \& Bent, T. (2008). Perceptual adaptation to non-native speech. Cognition, 106(2), 707-729. doi: 10.1016/j.cognition.2007.04.005

Clarke, C. M., \& Garrett, M. F. (2004). Rapid adaptation to foreign-accented English. Journal of the Acoustical Society of America, 116(6), 3647-3658. doi: 10.1121/1.1815131

Dahan, D., Drucker, S. J., \& Scarborough, R. A. (2008). Talker adaptation in speech perception: Adjusting the signal or the representations? Cognition, 108(3), 710718.

Eisner, F., Melinger, A., \& Weber, A. (2013). Constraints on the transfer of perceptual learning in accented speech. Frontiers in psychology, 4.

Farmer, T. A., Liu, R., Mehta, N. S., \& Zevin, J. D. (2009). Native language experience influences the perceived similarity of second language vowel categories. In Cogsci 2009: Proceedings of the 31st annual meeting of the cognitive science society (pp. 2588-2593).

Ito, K., \& Campbell-Kibler, K. (2011, August). Speaker-adaptation to /I/ - / $/$ / merger: An eye-tracking study. In Proceedings of the international congress of phonetic sciences xvii. Hong Kong.

Janse, E., \& Adank, P. (2012). Predicting foreign-accent adaptation in older adults. The Quarterly Journal of Experimental Psychology, 65(8), 1563-1585.

Kraljic, T., Samuel, A. G., \& Brennan, S. E. (2008). First impressions and last resorts: How listeners adjust to speaker variability. Psychological science, 19(4), 332-338.

Krestar, M., Incera, S., \& McLennan, C. T. (2013). Using mouse tracking to dxamine the time course of an auditory lexical decision task. Ohio Psychologist, 29 - 32.

Maye, J., Aslin, R. N., \& Tanenhaus, M. K. (2008). The weckud wetch of the wast: Lexical adaptation to a novel accent. Cognitive Science, 32(3), 543-562.

New, B., Pallier, C., Ferrand, L., \& Matos, R. (2001). Une base de données lexicales du français contemporain sur internet : LEXIQUE ${ }^{\mathrm{TM}}$. L'année psychologique, 101(34), 447-462.

Norris, D., McQueen, J. M., \& Cutler, A. (2003). Perceptual learning in speech. Cognitive psychology, 47(2), 204-238.

Pierrehumbert, J. (2002). Word-specific phonetics. Laboratory phonology VII. Berlin: Mouton de Gruyter, 101-40. 
Spivey, M. J., Grosjean, M., \& Knoblich, G. (2005). Continuous attraction toward phonological competitors. Proceedings of the National Academy of Sciences of the United States of America, 102(29), 10393-10398. doi: 10.1073/pnas.0503903102

Tanenhaus, M. K., Spivey-Knowlton, M. J., Eberhard, K. M., \& Sedivy, J. C. (1995). Integration of visual and linguistic information in spoken language comprehension. Science, 268(5217), 1632-1634. 


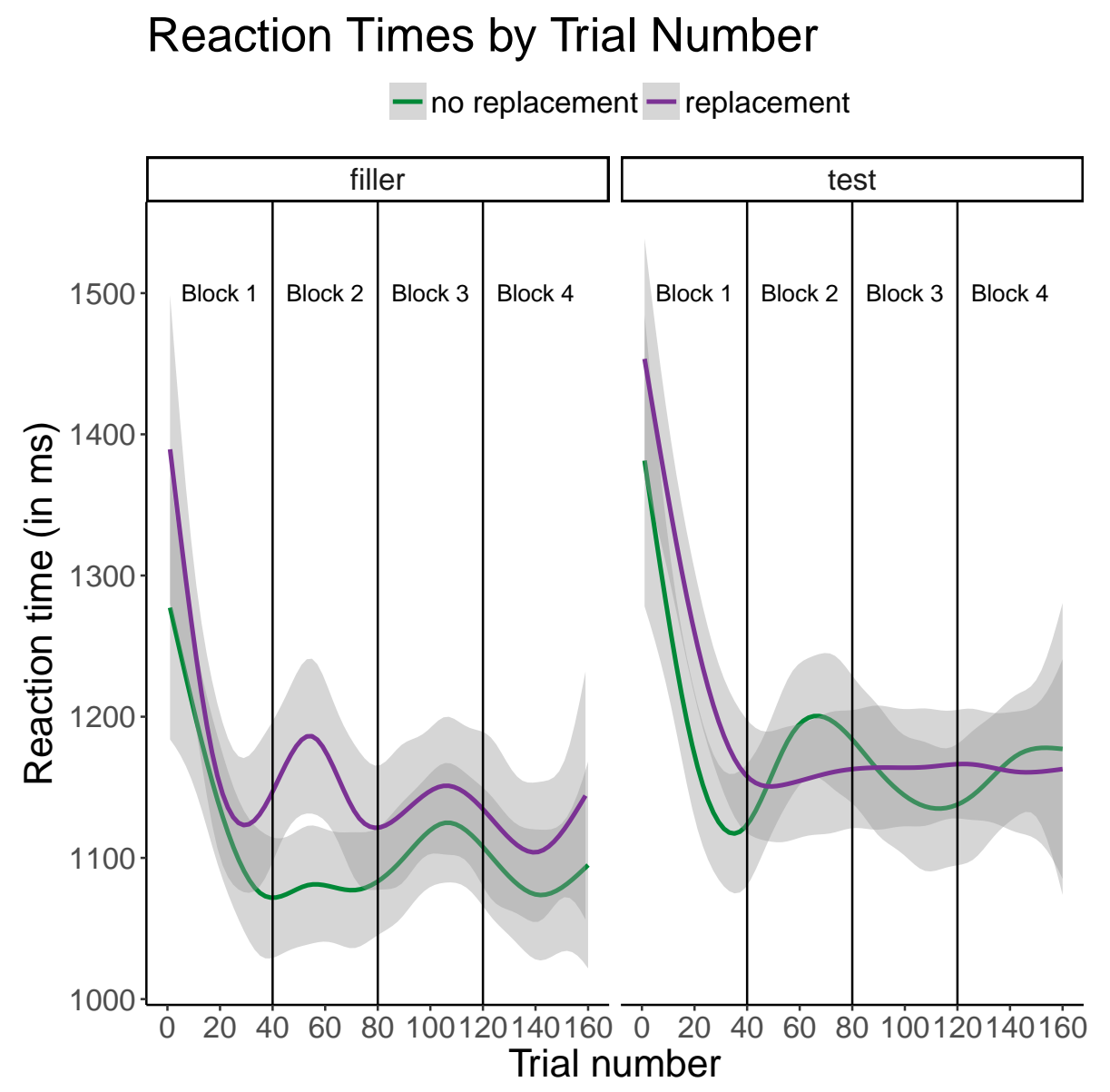

Figure 1: Reaction times in ms for filler and test items with no phoneme replacement and phoneme replacement items by trial number and block. 


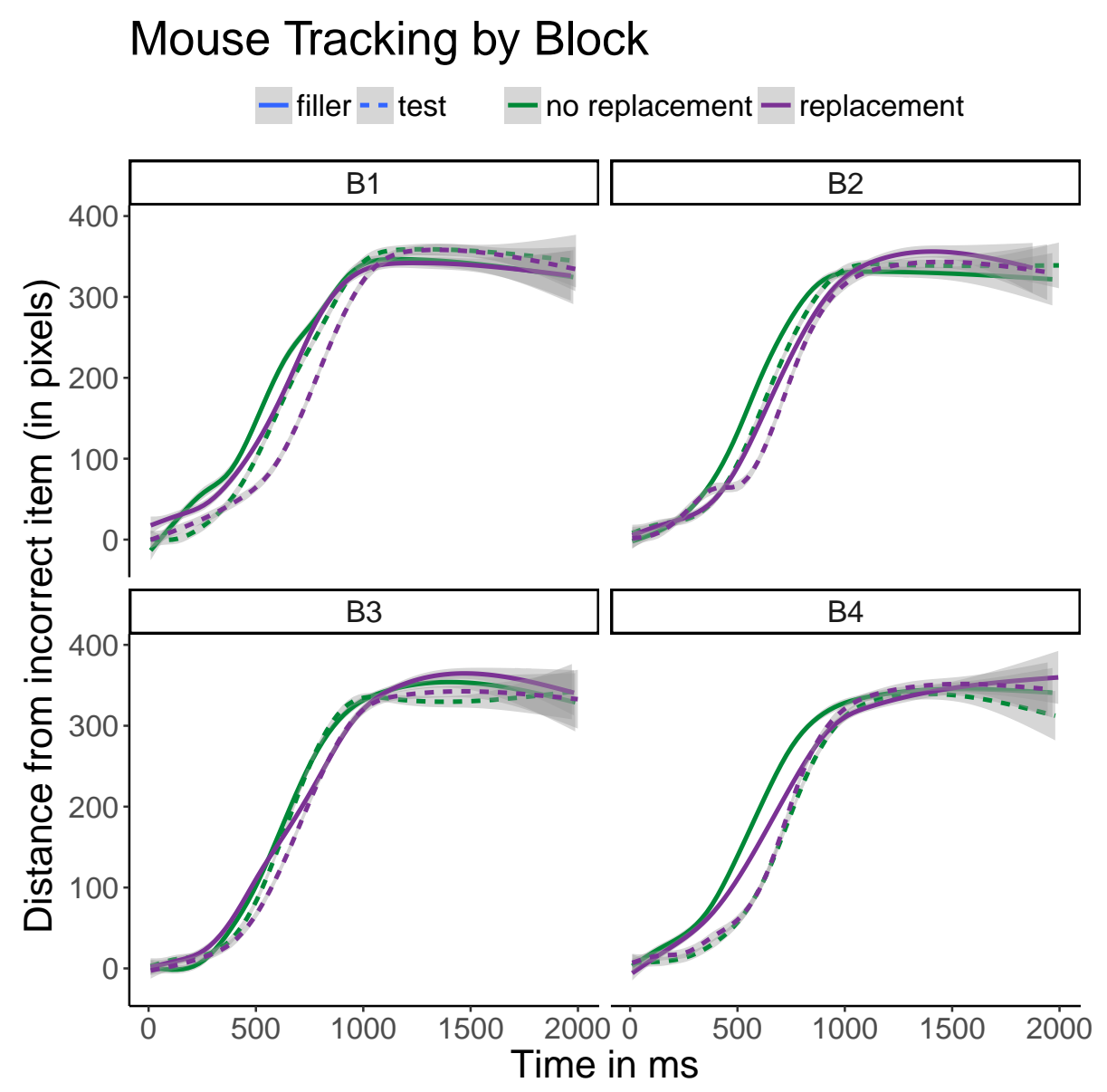

Figure 2: Mouse movement trajectories grouped by block, separated for filler and test, no replacement and replacement items. 


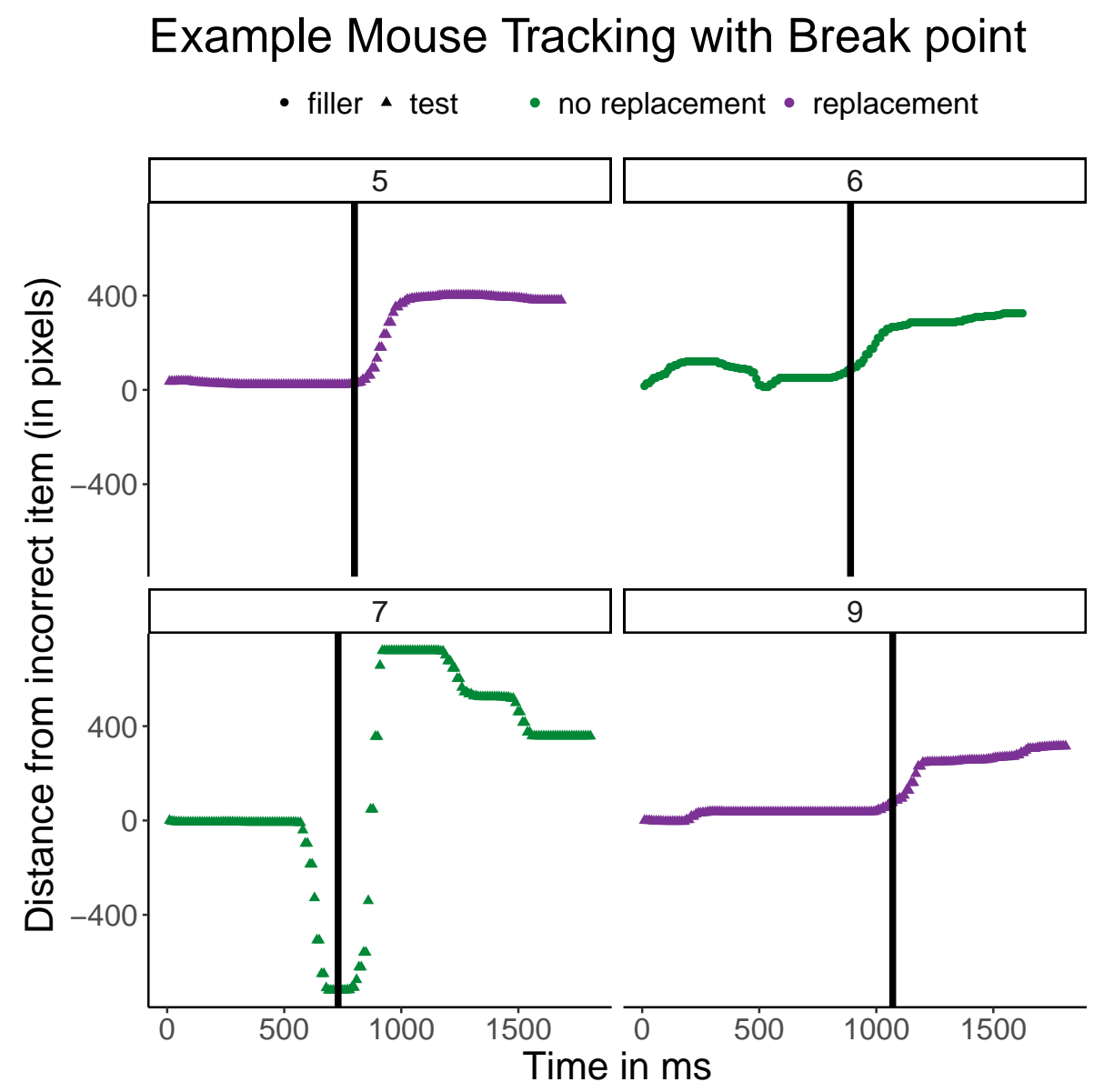

Figure 3: Examples of mouse movements with break points based on models. 


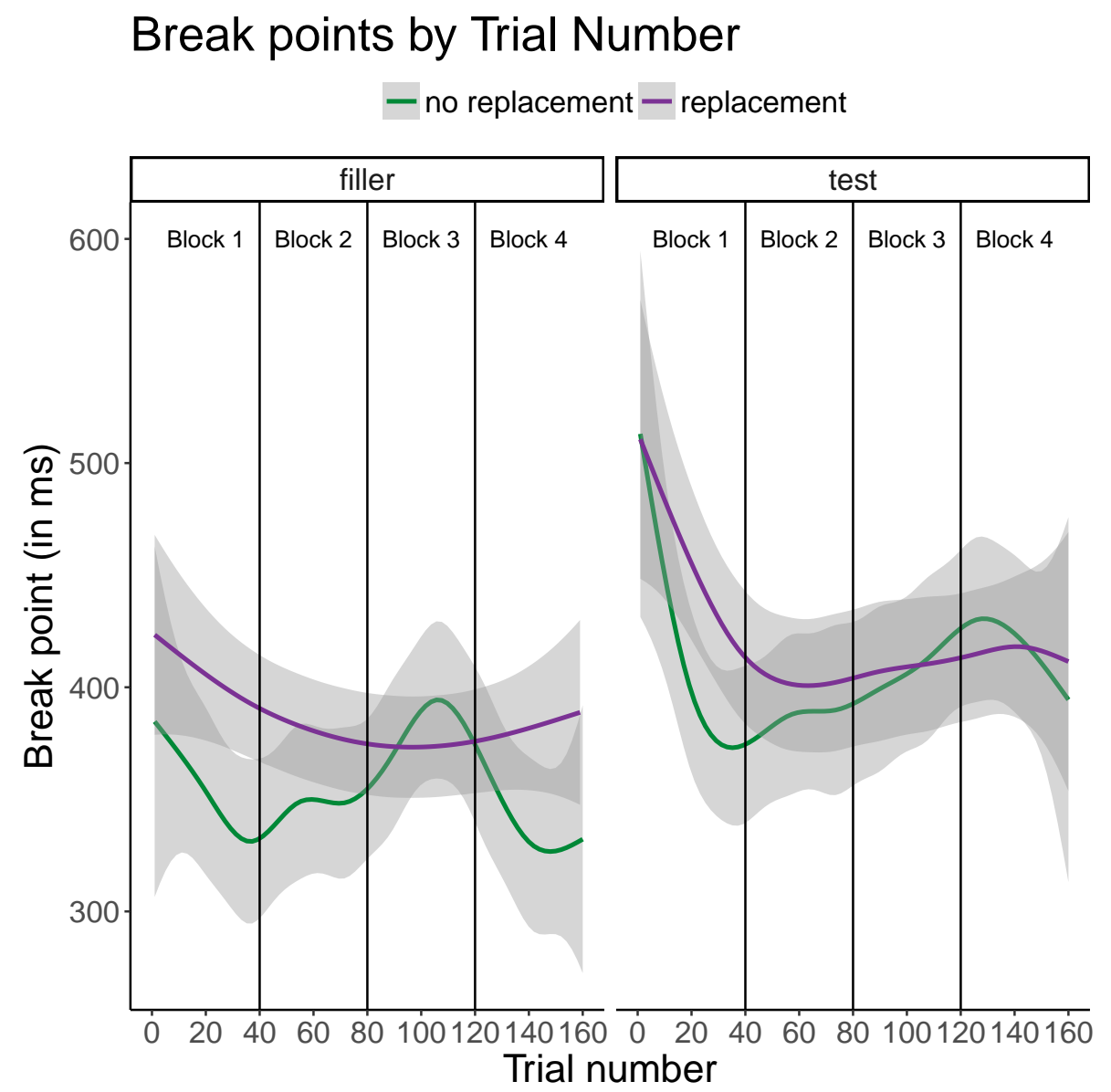

Figure 4: Mouse movement trajectories, grouped by block, for filler and test, no replacement and replacement, items. 